\title{
Relative Permeability Experiments of Carbon Dioxide Displacing Brine and Their Implications for Carbon Sequestration
}

\author{
Jonathan S. Levine, ${ }^{* \dagger, \S}$ David S. Goldberg, ${ }^{\ddagger}$ Klaus S. Lackner, ${ }^{\dagger}$ Juerg M. Matter, ${ }^{\ddagger} \|$ Michael G. Supp, ${ }^{\S}$ \\ and T. S. Ramakrishnan ${ }^{\S}$ \\ ${ }^{\dagger}$ Department of Earth and Environmental Engineering, Columbia University, New York, New York 10027, United States \\ ${ }^{\ddagger}$ Lamont Doherty Earth Observatory, Columbia University, Palisades, New York 10964, United States \\ ${ }^{\S}$ Schlumberger-Doll Research, Cambridge, Massachusetts 02143, United States
}

Supporting Information

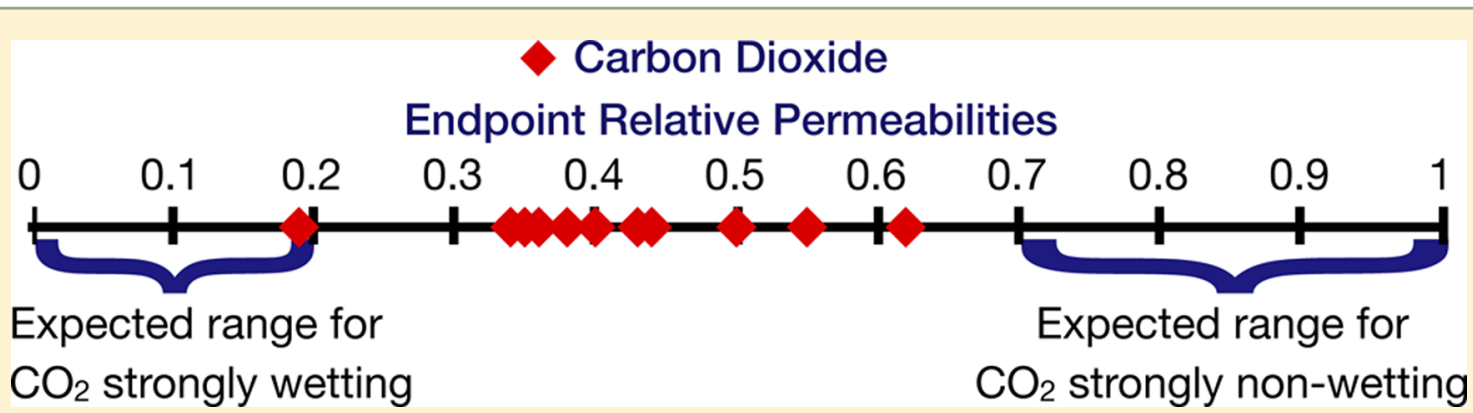

ABSTRACT: To mitigate anthropogenically induced climate change and ocean acidification, net carbon dioxide emissions to the atmosphere must be reduced. One proposed option is underground $\mathrm{CO}_{2}$ disposal. Large-scale injection of $\mathrm{CO}_{2}$ into the Earth's crust requires an understanding of the multiphase flow properties of high-pressure $\mathrm{CO}_{2}$ displacing brine. We present laboratory-scale core flooding experiments designed to measure $\mathrm{CO}_{2}$ endpoint relative permeability for $\mathrm{CO}_{2}$ displacing brine at in situ pressures, salinities, and temperatures. Endpoint drainage $\mathrm{CO}_{2}$ relative permeabilities for liquid and supercritical $\mathrm{CO}_{2}$ were found to be clustered around 0.4 for both the synthetic and natural media studied. These values indicate that relative to $\mathrm{CO}_{2}$, water may not be strongly wetting the solid surface. Based on these results, $\mathrm{CO}_{2}$ injectivity will be reduced and pressure-limited reservoirs will have reduced disposal capacity, though area-limited reservoirs may have increased capacity. Future reservoir-scale modeling efforts should incorporate sensitivity to relative permeability. Assuming applicability of the experimental results to other lithologies and that the majority of reservoirs are pressure limited, geologic carbon sequestration would require approximately twice the number of wells for the same injectivity.

\section{INTRODUCTION}

Carbon dioxide capture and sequestration has been proposed to alleviate carbon dioxide emissions from release into the atmosphere, typically by injection into the Earth's crust. ${ }^{1,2}$ Only a limited amount of $\mathrm{CO}_{2}$ can be disposed of in hydrocarbonbearing strata while increasing hydrocarbon production; therefore, large-scale geological $\mathrm{CO}_{2}$ disposal will require injection into brine-filled permeable strata. To maximize disposal capacities and minimize the risk of buoyancy-driven leakage through overlying impermeable strata, it is expected that these reservoirs will need to be at high pressures, i.e., large depths, to ensure high $\mathrm{CO}_{2}$ densities. Therefore, reservoir pressures are expected to be substantially larger than the $\mathrm{CO}_{2}$ critical pressure of $7.39 \mathrm{MPa}$. Because of the geothermal gradient, most reservoirs at the necessary pressures will be at temperatures greater than the $\mathrm{CO}_{2}$ critical temperature of $31.1^{\circ} \mathrm{C}$, though subseabed and subpermafrost reservoirs may be at lower temperatures at which $\mathrm{CO}_{2}$ would be liquid. Of particular interest are sediments ${ }^{3}$ and basalt ${ }^{4}$ underlying the deep ocean $\left(>2.7 \mathrm{~km}\right.$ depth) where liquid $\mathrm{CO}_{2}$ is denser than overlying ocean and pore waters and will be trapped by buoyancy. ${ }^{5}$ Thus, the reported set of experiments focuses on injection at a pressure, $10 \mathrm{MPa}$, equivalent to $1 \mathrm{~km}$ depth, that ensures a dense $\mathrm{CO}_{2}$ phase but is within the equipment limitations, with liquid $\mathrm{CO}_{2}$ experiments at $20{ }^{\circ} \mathrm{C}$ and supercritical $\mathrm{CO}_{2}$ experiments at $50{ }^{\circ} \mathrm{C}$.

Endpoint Relative Permeability. Neglecting body forces, Darcy's law as modified for multiphase flow ${ }^{6}$ defines relative permeability and is given by

$$
\mathbf{v}_{j}=-\frac{k k_{r j}\left(S_{j}\right)}{\mu_{j}} \nabla P_{j}
$$

where $k$ is the intrinsic permeability of the porous medium, and for each phase $j, \mathbf{v}_{j}$ is the velocity, $k_{r j}\left(S_{j}\right)$ is the relative

\section{Received: April 9, 2013}

Revised: November 18, 2013

Accepted: November 25, 2013

Published: November 25, 2013 
permeability at saturation $S_{j}, \mu_{j}$ is the shear coefficient of viscosity, and $P_{j}$ is the phase pressure. For two phase flow the phases are typically denoted by replacing $j$ with $w$ and $n$, i.e., the wetting and nonwetting phases, respectively. With two fluid phases saturating the porous medium $S_{w}=1-S_{n} . k_{r j}\left(S_{j}\right)$ accounts for the reduction in effective permeability for phase $j$ relative to the intrinsic permeability due to the presence of an additional phase. $k_{r j}$ varies between zero at residual saturation, $S_{j}^{o}$, defined as the saturation at which the fluid being displaced becomes disconnected and thus can no longer flow, and unity when no other phase is present, i.e. $k_{r j}\left(S_{j}^{o}\right)=0$, and $k_{r j}(1)=1$.

The maximum $k_{r j}$ for an invading fluid is limited by an immobile residual amount of the displaced fluid (subscript $d$ ) which stops flowing at its residual saturation, $S_{d}^{o}$ and is defined as the endpoint relative permeability, $k_{r j}^{o}=k_{r j}\left(S_{d}^{o}\right) . k_{r j}^{o}$ is determined by fluid occupancy at the pore and subpore scales due to capillarity. ${ }^{7,8} k_{r n}^{0}$ is typically in the $0.7-1$ range because the residual wetting phase remains at least on parts of the pore walls and in the smallest pores, blocking the smallest fluid pathways that least contribute to flow. Conversely, $k_{r w}^{o}$ is in the 0.1-0.3 range because the residual nonwetting phase blocks the largest pathways that most contribute to flow. I.e. $k_{r j}^{o}$ is measuring the multiphase fluid network that results from porescale fluid/fluid-rock surface chemistry interactions. Therefore, unlike measurements of $k_{r j}$ at lower intermediate saturations, $k_{r j}^{o}$ measures a fundamental fluid/fluid-rock property, providing a qualitative measure of in situ wettability. Furthermore, because capillarity determines both $k_{r j}^{o}$ and pore-scale fluid occupancy at lower $S_{j}$, the entire $k_{r j}$ curve is similarly scaled at lower $S_{j}{ }^{7,8}$ Numerical reservoir codes commonly model $k_{r j}$ with parametrized curves such as the modified Corey curve, with $k_{r j}\left(S_{j}\right)$ directly proportional to $k_{r j}^{o}{ }^{9-12}$ Ramakrishnan and Wasan ${ }^{13}$ provide a percolation theory justification of the relative permeability-saturation-capillary pressure relationship and the Brooks and Corey curve. Thus, our emphasis here is on the determination of the endpoint $\mathrm{CO}_{2}$ relative permeability, $k_{r c}^{o}$ measured at residual saturation of the displaced aqueous phase, $S_{a}^{o}$.

Relative Permeability and Carbon Sequestration. In analyzing the geological suitability of a reservoir for carbon sequestration several factors are of critical importance: $\mathrm{CO}_{2}$ injection rate, disposal capacity, and lateral and vertical containment, all of which are affected by relative permeability. The elevation of the injection pressure over the reservoir pressure is limited by the pressure at which fracturing occurs in either the target stratum or the overlying mechanical caprock, with a suitable safety factor. This fracturing pressure limit determines the maximum $\mathrm{CO}_{2}$ injection rate, and for pressurelimited reservoirs the disposal capacity, ${ }^{14}$ in direct proportion to relative permeability through eq 1 . Injectivity of $\mathrm{CO}_{2}$ also affects the areal spreading and migration of the $\mathrm{CO}_{2}$ plume, ${ }^{15,16}$ which may need to be laterally restricted due to, e.g., a finitearea overlying impermeable seal, the need to avoid leakage pathways, or area-limited pore space rights; see, e.g., ref 17 . More generally, the dynamics and therefore the migration of $\mathrm{CO}_{2}$ are dictated by the ratio of mobilities of the $\mathrm{CO}_{2}$ and aqueous phases (denoted by subscripts $c$ and $a$ ), $M \equiv \lambda_{c} / \lambda_{a}$ where the mobility of each phase is defined as $\lambda_{j}=k k_{r j} / \mu_{j}$ and incorporates all fluid-specific parameters in eq 1 . As a consequence, in addition to topographic and geological data, reservoir modeling for $\mathrm{CO}_{2}$ injection requires accurate multiphase flow experimental data, including the endpoint
$\mathrm{CO}_{2}$ relative permeability used to model $k_{r c}$ at lower saturations.

Previous experimental studies report maximum measured $k_{r c}$ outside of the 0.7-1 range expected if $\mathrm{CO}_{2}$ is strongly nonwetting relative to brine. ${ }^{18-28}$ In all cases $\mathrm{CO}_{2}$ did not imbibe without an applied pressure, i.e. capillary pressure $P_{C}>$ 0 , indicating that $\mathrm{CO}_{2}$ is not wetting or weakly wetting in the $\mathrm{CO}_{2}$-water system prior to $\mathrm{CO}_{2}$ contacting the surface. Many of these experiments do not correct for capillary end effects and buoyancy-induced fluid segregation, the lack of which may cause anomalously low values of $k_{r c}{ }^{7,8,11,29,30}$ Critically, none of these experiments present data to show that $P_{C}$ was sufficiently elevated to ensure $S_{a}^{o}$ was achieved. Because capillarity determines pore occupancy based on the smallest pore throat diameter, ${ }^{7,13,31,32}$ injecting many pore volumes at a constant $\mathrm{CO}_{2}$ volumetric flow rate, $Q_{c}$, at an insufficient $P_{C}$ will not achieve $S_{a}^{o}{ }^{7,8,11}$ Lower values of maximum $k_{r c}$ may therefore be the result of reduced flow path availability due to any of these three mechanisms, and without data showing that $S_{a}^{o}$ was achieved such relative permeability measurements cannot be considered measurements of endpoint $\mathrm{CO}_{2}$ relative permeability. [Note that some of these articles explicitly state these points and provide data and discussions of these phenomena.] Further details are contained in the Supporting Information.

Pini and Benson ${ }^{33}$ presented very similar experiments to those previously performed in this study. ${ }^{34} k_{r o}^{o}, P_{C}$, and $S_{a}$ were measured following the method developed in Ramakrishnan and Cappiello, ${ }^{35}$ which stated that $S_{a}$ could be determined using mass balance, nuclear magnetic resonance imaging (NMR), or X-ray computerized tomographic imaging (X-ray CT). Fordham et al. ${ }^{36}$ and Pini et al. ${ }^{37}$ demonstrated results with NMR and X-ray CT, respectively. Fordham et al. ${ }^{36}$ specifically used NMR imaging with calibrated volume chambers to unambiguously determine saturation profiles using a heavy water-dodecane system and clearly showed the presence of capillary end effects. Pini and Benson ${ }^{33}$ used the technique developed in ref 35 , taking the derivative of a polynomial fit to the data rather than a derivative of the data or the function suggested by ref 35 . Based on the derivative of the polynomial, they report $k_{r c}^{o}=0.32$ and 0.92 for gaseous $\mathrm{CO}_{2} /$ brine and supercritical $\mathrm{CO}_{2} /$ brine, respectively, and conclude that $\mathrm{CO}_{2}$ is strongly nonwetting relative to brine. However, measuring endpoint relative permeability using the technique of ref 35 requires identifying a linear slope of pressure vs flow-rate occurring at several times the entry capillary pressure. More complex data fitting requires carefully choosing a functional form that asymptotes to a straight line. Using only the linear portion of these data (see Figure $2 \mathrm{a}$ ), we find that $k_{r c}^{o}=0.28$ and 0.57 for the gaseous $\mathrm{CO}_{2}$ and supercritical $\mathrm{CO}_{2}$ experiments, respectively, indicating that water may not be strongly wetting in a $\mathrm{CO}_{2}$-water system. Furthermore, their data show higher $k_{r c}^{o}$ values for supercritical $\mathrm{CO}_{2}$ than gaseous $\mathrm{CO}_{2}$, different from what we observe.

In the carbon sequestration literature two common assumptions are followed: (1) the relative permeabilities of the $\mathrm{CO}_{2}-\mathrm{H}_{2} \mathrm{O}$ system are that of a $\mathrm{CO}_{2}$-nonwetting/waterwetting system, and (2) reactions with minerals are driven by the species dissolved in the aqueous phase. The second assumption relies on the ability of the aqueous phase to be in full contact with the grain surface, implying a strongly waterwet system. Previous $k_{r c}$ studies reported relatively low maximum $k_{r c}$ values indicative of a weakly water wetting system. However, with one exception, $k_{r c}^{o}$ was not directly 


\section{High Pressure Core Flooding Reactor}

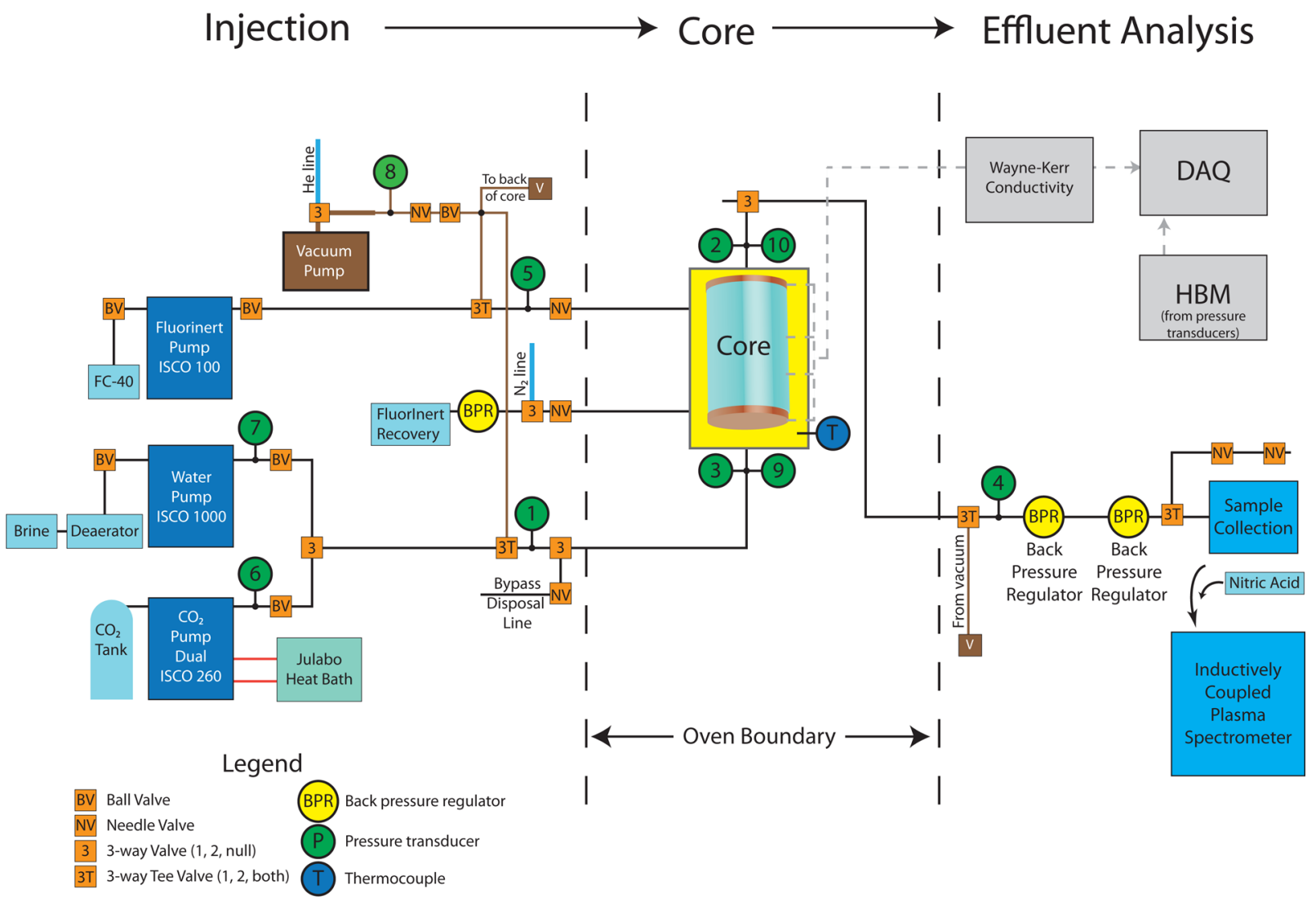

Figure 1. The core flooding system; 1 and $20 \mathrm{MPa}$ transducers are colocated before and after the core. Abbreviations: DAQ = Data Analysis Computer; HBM $=$ HBM pressure signal measurement system.

identified in these studies as per the discussion above. Therefore, we conducted a series of flow-through experiments specifically designed to measure $k_{r c}^{o}$ in the $\mathrm{CO}_{2}$-water system. Measurement of $k_{r c}^{o}$ provides both a qualitative measure of wettability, i.e. pore-scale surface chemistry, as well as a direct measure of the resultant chemistry-induced multiphase fluid conductivity. Due to capillarity-controlled pore-scale fluid occupancy, $k_{r c}$ at all saturations will scale with $k_{r c}^{o}$. If $\mathrm{CO}_{2}$ is strongly nonwetting relative to brine, drainage endpoint $\mathrm{CO}_{2}$ relative permeability would be expected to be in the range of $0.7-1$.

\section{MATERIALS AND METHODS}

A schematic of the experimental system developed in ref 34 is shown in Figure 1. Before each experiment, air was evacuated from the system using a two-stage vacuum pump, and deaerated brine was used to saturate the rock. The brine was constituted by mixing $18.2 \mathrm{M} \Omega \mathrm{cm}$ ultrapure water (Millipore Synergy UV) with Fisher Scientific ACS grade $\mathrm{NaCl}$ and $\mathrm{LiCl}$ salts at a 1000:1 ratio by mass, with $\mathrm{LiCl}$ included as a tracer for salt. A two-cylinder piston pump (Isco) for liquid and supercritical $\mathrm{CO}_{2}$ and a dual reciprocating near pulseless pump (Quizix) for gas phase injection were used to inject $\mathrm{CO}_{2}$ to displace brine. $\mathrm{CO}_{2}$ was $99.8 \%$ dry (American Gas Products). The core was held vertically in a core holder (Temco) with $\mathrm{CO}_{2}$ typically displacing brine from the top, though experiments were performed in both directions. Radial stress was applied on a rubber sleeve by pumping Fluorinert $(3 \mathrm{M})$ into the surrounding chamber at a controlled pressure, typically 13.7 MPa. Pressure transducers (HBM, 18-bit processing accuracy, $1 \mathrm{MPa}$ : $0.1 \%$ full-scale accuracy, 20 MPa: $0.2 \%$ full-scale accuracy, simultaneously calibrated with a primary standard dead weight tester with 5 ppm accuracy) were colocated at either end of the core holder to measure the pressure drop across the core for permeability measurements. The core holder and pressure transducers were contained in a temperature-controlled chamber (Watlow) to maintain a constant temperature during each experiment. Four impedance terminals were connected to two current plates that also serve as a fluid distributor and two voltage terminals embedded within the rubber boot. A rough measure of average brine saturation between the voltage terminals could be obtained from resistance data. A pair of back pressure regulators (Tescom) were used to control pressure at the core exit, which in most of the experiments was $10 \mathrm{MPa}$. The effluent brine and $\mathrm{CO}_{2}$ were collected in pressurized sampling containers (Swagelok) to provide the average brine saturation in the core by mass balance, which is used to calculate $S_{a}^{o}$ (see eq 3 below). All equipment was monitored and controlled by a data acquisition system with a custom-written National Instruments LabView program. $\mathrm{CO}_{2}$ physical data used in experimental calculations are from NIST, ${ }^{38}$ while brine physical data are from ref 39. 
Table 1. Endpoint $\mathrm{CO}_{2}$ Relative Permeability and Average Saturation: Experimental Conditions and Results

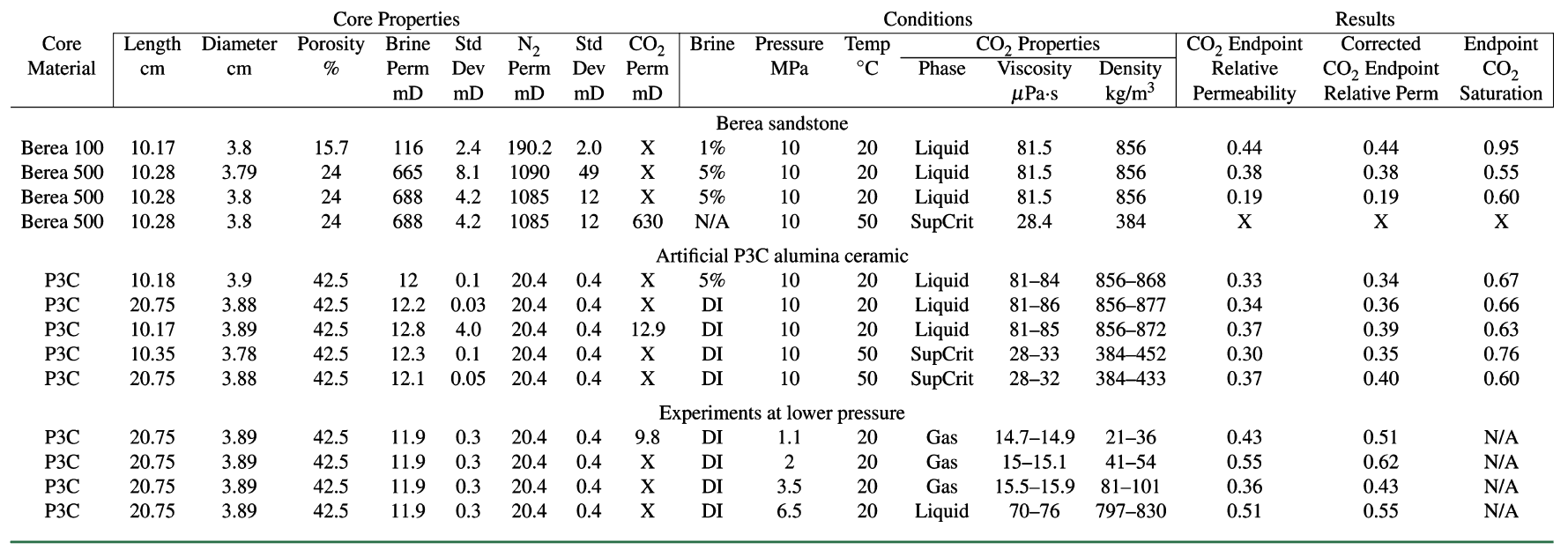

Cores included Berea sandstone, chosen for its relative homogeneity, and synthetic P3C alumina ceramic (CoorsTek), which is inert to brine, $\mathrm{CO}_{2}$, and carbonic acid. Cores were prepared to be approximately $4^{\prime \prime}$ in length and $1.5^{\prime \prime}$ in diameter, with the exact dimensions used in calculations. Later experiments were conducted with 8 " length P3C cores to minimize capillary end effects. All cores were vacuum-dried overnight at $70{ }^{\circ} \mathrm{C}$ prior to each experiment. Helium intrusion porosity measurements were made on cores that were $1.5^{\prime \prime}$ long and $1.5^{\prime \prime}$ diameter and cored from rock adjacent to the cores used in the experiment. Mercury intrusion porosimetry was previously performed on cores from similar samples and the resulting pore size distribution data used to obtain $S_{a}^{o}$ (see below).

Brine permeability was measured at the beginning of each experiment at both atmospheric and experimental pressures by flowing brine at a series of increasing flow rates followed by an identical series of decreasing steps in flow rate at evenly spaced increments, typically $0-20 \mathrm{~mL} / \mathrm{min}$ in $4 \mathrm{~mL} / \mathrm{min}$ increments. Permeability was calculated from a least-squares linear fit to the slope of volumetric flow rate vs pressure drop across the core, $\Delta P_{i o} \equiv P_{i}-P_{o}$, where subscripts $i$ and $o$ denote conditions at the core inlet and outlet, respectively. At least three independent measurements of permeability were made, and their average was reported for each experiment, with similar values at atmospheric and experimental pressures. $\mathrm{CO}_{2}$ permeability was measured on three cores following the same procedures as those used to measure brine permeability. Brine and $\mathrm{CO}_{2}$ permeability agreed within variations between independent measurements (see Table 1) given the limits of the equipment and the small $\Delta P_{i o}$ during $\mathrm{CO}_{2}$ permeability measurements.

The endpoint $\mathrm{CO}_{2}$ relative permeability experiments presented here use the technique proposed by ramakrishnan and Cappiello. ${ }^{35}$ The advantage of this method is that capillary end effects are automatically removed by considering differential increases in flow rate with respect to $\Delta P_{i o}$ or equivalently injection pressure. Although the entire $k_{r n}$ curve may be obtained with this method, our emphasis here is the determination of $k_{r c}^{o}$. This amounts to obtaining the increase in $\Delta P_{i o}$ due to an imposed increase in steady-state volumetric flow rate of $\mathrm{CO}_{2}\left(Q_{c}\right)$, at inlet saturations close to $S_{a}^{o}$. Pressure in both phases just outside the outlet of the core must be equal to the wetting phase pressure, here the aqueous phase, in the pores in the outlet face of the core. At steady state $Q_{a}=0$ and so, from eq $1, P_{a}$ must be constant throughout the core, and thus $P_{a}=P_{o}$, the nominal pressure, throughout the core. At the core inlet, $P_{C i}=P_{i}-P_{a}=P_{i}-P_{o}=\Delta P_{i o}$. The solution for $k_{r c}=$ $\hat{k}_{r c}\left(P_{C i}\right)$ and $S_{a}=\hat{S}_{a}\left(P_{C i}\right)$ as functions (denoted by $\left.{ }^{\wedge}\right)$ of inlet capillary pressure, with $P_{C i}=\Delta P_{i o}$ are

$$
\begin{aligned}
& \hat{k}_{r c}\left(P_{C i}\right)=\frac{L}{A} \frac{\mu_{c}}{k} \frac{\mathrm{d} Q_{c}}{\mathrm{~d} \Delta P_{i o}} \\
& \hat{S}_{a}\left(P_{C i}\right)=\frac{L}{A} \frac{\mu_{c}}{k k_{r c}\left(P_{C i}\right)} \frac{\mathrm{d}\left(Q_{c} \bar{S}_{a}\right)}{\mathrm{d} \Delta P_{i o}}
\end{aligned}
$$

where $L$ is core length, $A$ is core area, and $\bar{S}_{a}$ is the average brine saturation, determined here by effluent mass balance at each steady-state injection rate. Figure 2 shows pressure drop vs flow rate for each experiment, the local slope of which is used to determine $k_{r c}^{o}$.

Although mass flux is constant at steady state, volumetric flux increases as pressure decreases from the inlet to the outlet end of the porous medium. Therefore, $k_{r c}$ obtained by the method of ref 35 must be corrected for this volumetric compressibility effect. ${ }^{40}$ For an injected steady-state mass flow rate of $\mathrm{CO}_{2}, \dot{m}_{i}$, at each $P_{i}$

$$
\hat{k}_{r c}\left(P_{C i}\right)=\frac{L}{A} \frac{\mu_{c}\left(P_{i}\right)}{k \rho_{c}\left(P_{i}\right)} \frac{\mathrm{d} \dot{m}_{i}}{\mathrm{~d} \Delta P_{i o}}
$$

where $\rho_{c}\left(P_{i}\right)$ is the density at $P_{i}$. While the correction is small for liquid $\mathrm{CO}_{2}$, it is nontrivial for gaseous and supercritical $\mathrm{CO}_{2}$, see Table 1 below.

Due to capillarity, achieving flow across a core requires a minimum $P_{i}$, called the breakthrough pressure $P_{b}$, to overcome the $P_{C}$ for the largest set of pore throat radii that allows for a single flow path across the core. From the Young-Laplace equation, $P_{C}$ is inversely proportional to pore throat radius, and so increases in $P_{C}$ relative to $P_{b}$ will cause the nonwetting $\mathrm{CO}_{2}$ to invade pores connected by correspondingly decreasing pore throat radii. Therefore, $S_{a}^{o}$ was achieved by raising $\Delta P_{i o}$ above the independently measured $P_{b}{ }^{35}$ by at minimum the ratio of the largest to smallest pore throats as measured by $\mathrm{Hg}$ intrusion porosimetry (Figures S1 and S2). Establishment of a constant value of $d Q_{c} / d \Delta P_{i o}$, i.e. a linear slope, at high flow rates confirms that capillarity-determined flow paths, and equivalently pore occupancy, are unchanged and thus that $S_{a}^{o}$ was reached at the inlet.

With $\gamma$ as the interfacial tension between the two fluids, the capillary number $N_{C}=v_{c} \mu_{c} / \gamma$ was about $10^{-8}$ for these experiments, far below the residual wetting phase mobilization 

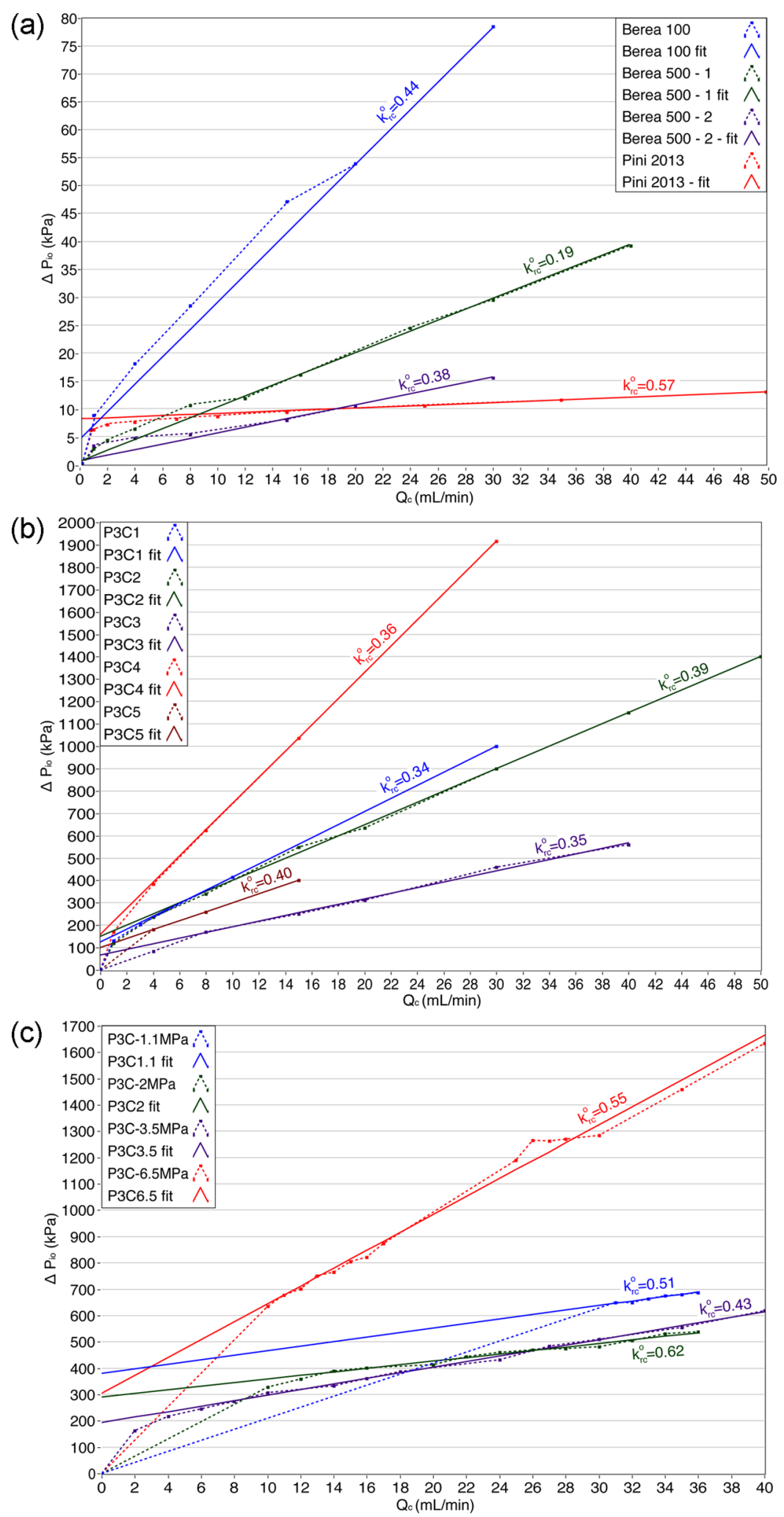

Figure 2. Measured pressure difference as a function of flow rate for (a) Berea sandstone experiments including comparable data from ref 33, (b) P3C experiments at $10 \mathrm{MPa}$, and (c) P3C experiments at lower pressures indicated in the figure legend. The local slope is used to compute endpoint $\mathrm{CO}_{2}$ relative permeability. See Table 1 for detailed results; experiments correspond to the labeled $k_{r c}^{o}$ values shown in the figures.

threshold of about $10^{-3} \cdot{ }^{41}$ Capillarity dominates during core scale $\mathrm{CO}_{2}$ /brine experiments, stabilizing viscous instabilities. ${ }^{42}$ Nonetheless, for the sake of robustness initial experiments injected $\mathrm{CO}_{2}$ into the core from below so that buoyancy and viscous instabilities were reinforced, while later experiments injected $\mathrm{CO}_{2}$ from above and confirm that the results were

largely unchanged. 


\section{RESULTS AND DISCUSSION}

Experimental conditions and results are reported in Table 1, ordered first by core material, then by pressure and temperature and hence $\mathrm{CO}_{2}$ state. Figure 2 shows the determination of $k_{r c}^{\circ}$ from the linear slope of $\Delta P_{i o}$ vs $Q_{c}$ at the highest flow rates. $Q_{c}$ $\Delta P_{i o}$, and $\bar{S}_{c}$ data are available in the Supporting Information. $k_{r c}^{o}$ was found to have similar intermediate values at all conditions, ranging from 0.19 to 0.62 , but with the majority of results clustered between 0.34 and 0.44 . The characteristic convex shape of the plot of $\Delta P_{i o}$ vs $Q_{c}$ as well as $P_{C}>0$ are indicative of a nonwetting phase intrusion, confirming that $\mathrm{CO}_{2}$ is the nonwetting phase in comparison to water. Endpoint $\mathrm{CO}_{2}$ saturation, defined as $1-S_{a}^{o}$, was determined from $\bar{S}_{a}$ measured by mass balance and processed using eq 3 and varied from 0.55 to 0.95 during dense-phase experiments. Impedance-derived saturation measurements (Figures S3 and S4) on the first several cores confirmed the low brine saturations measured from mass balance.

To confirm that the measurements of $k_{r c}^{o}$ were measuring the desired multiphase flow effects and not an experimental artifact, a number of experimental variations were performed, each designed to confirm that a particular potential source of fundamental experimental design error was not affecting the results. While the earliest experiments were run with a $5 \% \mathrm{NaCl}$ brine to simulate in situ salinities, later experiments were run with no or low salt to ensure that salt precipitation could not have reduced the intrinsic rock permeability. Experiments were run at gaseous, liquid, and supercritical $\mathrm{CO}_{2}$ conditions to probe the effect of $\mathrm{CO}_{2}$ state and to simulate different reservoir conditions. Similarly, to ensure an appropriate baseline permeability for the relative permeability experiments, measurements were made for gaseous, liquid, and supercritical $\mathrm{CO}_{2}$ permeability on a subset of cores and confirmed that $\mathrm{CO}_{2}$ permeabilities are in agreement with liquid water permeability (compare brine, $\mathrm{CO}_{2}$, and $\mathrm{N}_{2}$ permeabilities in Table 1). To confirm that the relative permeability method handled capillary end effects correctly, the final six P3C experiments used longer 8 " cores with relatively small capillary end effects, with the results substantially the same.

Prior to these experiments, it was anticipated that $\mathrm{CO}_{2}$ would behave as a strongly nonwetting fluid and that $\mathrm{CO}_{2}$ drainage endpoint relative permeabilities would therefore be in the range of $0.7-1$. Instead, the experiments yielded intermediate values of $k_{r o}^{o}$ clustered around $0.35-0.4$. The median was 0.39 and the mean was 0.41 with a standard deviation of 0.11 , while densephase experiments had median and mean $k_{r c}^{o}$ of 0.38 with a standard deviation of 0.09 . High endpoint $\mathrm{CO}_{2}$ saturations (Berea: 0.55, 0.6, 0.95, P3C: 0.6-0.76) were consistent with typical values for $S_{a}^{o}$ from the theoretical literature and were higher than most previously reported saturations from the $\mathrm{CO}_{2}$ literature, e.g., refs 18-21 with the lowest measurement in agreement with more recent measurements of $S_{a}^{o} 33$ and $S_{a}$ close to $S_{a}^{o}{ }^{26,27}$ While the results might appear to be independent of system conditions, with perhaps the exception of the gas phase results, such a conclusion would require a more systematic set of experiments to probe sensitivity to each variable.

$\mathrm{H}_{2} \mathrm{O}$ is slightly soluble in dense-phase $\mathrm{CO}_{2}$, and, therefore, residual water will evaporate into the flowing $\mathrm{CO}_{2}$, slowly increasing $k_{r c}{ }^{17}$ Our results during the extended $\mathrm{CO}_{2}$ flowing period did not indicate a gradual increase in $k_{r c}$, probably because of the relatively short time-scale of each experiment. Berg et al. ${ }^{28}$ found negligible differences in $\bar{S}_{a}$ between unsaturated and saturated drainage experiments due to the limited solubility of water into $\mathrm{CO}_{2}$. However, they also report subcore scale differences in saturation profiles, though with too many experimental design differences to be able to draw any conclusions with respect to this work.

Water evaporation may also cause salt precipitation. Since salt occupies a fraction of the volume occupied by water (at most $2 \%$ in our experiments), and its precipitation is likely to occur on the trailing edge of the displacement front, we do not expect halite formation to be the cause of the reduction in endpoint permeability. This is further confirmed by the experiments conducted with deionized water in synthetic media with little difference in the results between $5 \%$ salt and deionized water. Reduced intrinsic permeability due to chemical rearrangement of the pore network cannot explain the results as both the synthetic alumina and sandstone cores studied are relatively inert and further reinforced by results that are similar in both media.

Our experimental measurements suggest endpoint $\mathrm{CO}_{2}$ relative permeabilities that are smaller by about a factor of 2 than those expected for a strongly water-wetting system and are possibly reflective of a weakly water-wetting system, especially when dense $\mathrm{CO}_{2}$ is the injected fluid. When the same experiment was conducted with $\mathrm{N}_{2}$, an endpoint relative permeability closer to unity was obtained. Further experiments are necessary to confirm or refute the wettability hypothesis, which should include more complex lithologies than the relatively homogeneous and inert synthetic P3C alumina and Berea sandstone cores studied here.

The chemistry of $\mathrm{CO}_{2}$, brine, and mineral surfaces has proven to be complex. Typically low contact angles, in the 20$40^{\circ}$ range, ${ }^{43-45}$ conflict with reports of higher contact angles in the $50-70^{\circ}$ range at higher pressures and ionic strengths. ${ }^{46}$ Flow-through experiments in glass micromodels suggest that $\mathrm{CO}_{2}-\mathrm{H}_{2} \mathrm{O}$ systems may be weakly water wetting or intermediate wetting at pressures and temperatures similar to those in this study. ${ }^{47,48}$ Similarly, molecular simulations show aqueous phase $\mathrm{CO}_{2}$ reactions driving surface reactivity, ${ }^{49}$ i.e. $\mathrm{CO}_{2}$ does not simply behave as an inert fluid and alters the system wettability. The results here point to the continuing need for further pore- and molecular-scale experimental data on transport and surface chemistry processes, with a particular need to understand the dynamics of $\mathrm{CO}_{2}$ and thin brine films, e.g., ref 50 .

Implications for Geologic Carbon Sequestration. For a fixed injection pressure, halving the endpoint $\mathrm{CO}_{2}$ relative permeability from the expected range of $0.7-1$ will cause a proportional decrease in $\mathrm{CO}_{2}$ injectivity, discussed above and also shown by displacement models. ${ }^{17} \mathrm{CO}_{2}$ reservoirs limited by the fracturing pressure of the overlying caprock, e.g., refs 14 and 51, will have decreased disposal capacity. By contrast, because plume spreading occurs with respect to a characteristic migration advective velocity which is directly proportional to $\lambda_{c}{ }^{16} \mathrm{CO}_{2}$ reservoirs limited by areal extent will have increased disposal capacity. A reduction in $\mathrm{CO}_{2}$ mobility may also stabilize plume fronts, as similar analyses have shown for water flooding in oil reservoirs. ${ }^{7}$ A variety of indirect effects, including plume evolution and residual capillary trapping, may also be linked to mobility. 52

Future petrophysical analyses for potential $\mathrm{CO}_{2}$ sequestration sites should include relative permeability measurements on reservoir core samples rather than relying on extrapolations based on prior studies. Based on these results, $\mathrm{CO}_{2}$ injectivity 
will be reduced and pressure-limited reservoirs will have reduced disposal capacity, though area-limited reservoirs may have increased capacity. Assuming the majority of reservoirs are pressure limited, and if the experimental results reported here are found to apply to other lithologies as well, geologic carbon sequestration at scale will require about twice the number of disposal sites and/or wells.

\section{ASSOCIATED CONTENT}

\section{(S Supporting Information}

Tabulated numerical results for each experiment $\left(Q_{c}, \Delta P_{i o}, \bar{S}_{a}\right)$, mercury intrusion porosimetry data, and resistivity-derived local saturation data. This material is available free of charge via the Internet at http://pubs.acs.org.

\section{AUTHOR INFORMATION}

\section{Corresponding Author}

*E-mail: jonathan.levine@netl.doe.gov. Phone: 412-386-7534. Fax: 412-386-4604.

\section{Present Addresses}

${ }^{\S}$ National Energy Technology Laboratory, Pittsburgh, PA 15236.

"Ocean and Earth Science, University of Southampton, UK.

\section{Notes}

The authors declare the following competing financial interest(s): Schlumberger has a carbon service business.

\section{ACKNOWLEDGMENTS}

J.L. acknowledges the entire $\mathrm{CO}_{2}$ research group at Schlumberger-Doll Research for help with experiments as well as helpful discussions. This work was supported by a NYSERDA grant (NYSERDA-10113), and J.L. acknowledges support from the U.S. National Science Foundation, through a Fellowship in the IGERT Joint Program in Applied Mathematics and Earth and Environmental Science at Columbia University. The manuscript was greatly improved thanks to the comments and feedback received from the reviewers.

\section{NOMENCLATURE}

$k \quad$ permeability

$\hat{k}_{r j}\left(S_{j}\right)$ relative permeability of phase $j$, which is a function of the saturation of phase $j$

$k_{r j}^{o} \quad$ endpoint relative permeability of phase $j$

$\dot{m}_{j} \quad$ mass flow rate of phase $j$

$v_{j} \quad$ velocity of phase $j$

A area

$L \quad$ length

$N_{C} \quad$ capillary number

$\Delta P_{\text {io }} \quad$ pressure difference between the inlet and outlet of the core

$P_{b} \quad$ breakthrough pressure

$P_{C} \quad$ capillary pressure

$P_{j} \quad$ pressure, phase $j$ or location: $i=$ inlet, $o=$ outlet

$Q_{j} \quad$ volumetric flow rate of phase $j$

$\bar{S}_{j} \quad$ average saturation of phase $j$

$S_{j} \quad$ saturation of phase $j$

$S_{j}^{o} \quad$ residual saturation of phase $j$

$\gamma \quad$ interfacial tension

$\lambda_{j} \quad$ mobility of phase $j ; \lambda_{j}=k k_{r j} / \mu_{j}$

$\mu_{j} \quad$ shear coefficient of viscosity of phase $j$

$\rho_{j} \quad$ density of phase $j$

\section{Subscripts}

a aqueous phase

$c \mathrm{CO}_{2}$ phase

$C$ capillary, either capillary pressure or capillary number

$d$ phase being displaced

$i$ core inlet

$j$ phase $j$

$n$ nonwetting phase

$o$ core outlet

$w$ wetting phase

\section{REFERENCES}

(1) Fuels decarbonization and carbon sequestration: report of a workshop; Socolow, R., Ed.; 1997.

(2) IPCC Special Report on Carbon Dioxide Capture and Storage; Metz, B., Davidson, O., de Coninck, H., Loos, M., Meyer, L., Eds.; 2005.

(3) Levine, J. S.; Matter, J. M.; Goldberg, D. S.; Cook, A.; Lackner, K. S. Gravitational trapping of carbon dioxide in deep sea sediments: Permeability, buoyancy, and geomechanical analysis. Geophys. Res. Lett. 2007, 34.

(4) Goldberg, D. S.; Takahashi, T.; Slagle, A. L. Carbon dioxide sequestration in deep-sea basalt. Proc. Natl. Acad. Sci. 2008, 105, 9920.

(5) Koide, H.; Shindo, Y.; Tazaki, Y.; Iijima, M.; Ito, K.; Kimura, N.; Omata, K. Deep sub-seabed disposal of $\mathrm{CO} 2-$ The most protective storage. Energy Convers. Manage. 1997, 38, 253-258.

(6) Leverett, M. C. Flow of oil-water mixtures through unconsolidated sands. Trans. AIME 1939, 132, 149-171.

(7) Willhite, G. P. Waterflooding; Society of Petroleum Engineers: Richardson, TX, 1986.

(8) Anderson, W. Wettability literature survey part 5: The effects of wettability on relative permeability. J. Pet. Technol. 1987, 39, 14531468.

(9) Corey, A. T. The interrelation between gas and oil relative permeabilities. Prod. Mon. 1954, 19, 38-41.

(10) Land, C. Calculation of imbibition relative permeability for twoand three-phase flow from rock properties. SPE J. 1968, 8, 149-156.

(11) Honarpour, M.; Koederitz, L.; Harvey, A. Relative permeability of petroleum reservoirs; CRC Press Inc.: Boca Raton, FL, 1986.

(12) Lake, L. W. Enhanced Oil Recovery; Prentice Hall: 1989.

(13) Ramakrishnan, T. S.; Wasan, D. T. Effect of capillary number on the relative permeability function for two-phase flow in porous media. Powder Technol. 1986, 48, 99-124.

(14) Lucier, A.; Zoback, M.; Gupta, N.; Ramakrishnan, T. S. Geomechanical aspects of $\mathrm{CO} 2$ sequestration in a deep saline reservoir in the Ohio River Valley region. Environ. Geosci. 2006, 13, 85.

(15) Hesse, M. A.; Orr, F. M.; Tchelepi, H. A. Gravity currents with residual trapping. J. Fluid Mech. 2008, 611, 35-60.

(16) de Loubens, R.; Ramakrishnan, T. S. Analysis and computation of gravity induced migration in porous media. J. Fluid Mech. 2011, 675, $60-86$.

(17) Kopp, A.; Class, H.; Helmig, R. Investigations on CO2 storage capacity in saline aquifers-Part 2: Estimation of storage capacity coefficients. Int. J. Greenhouse Gas Control 2009, 3, 277-287.

(18) Bachu, S.; Bennion, B. Effects of in situ conditions on relative permeability characteristics of CO2-brine systems. Environ. Geol. 2008, 54, 1707-1722.

(19) Bennion, B.; Bachu, S. Drainage and imbibition CO2/brine relative permeability curves at reservoir conditions for carbonate formations, SPE 134028. SPE Annual technical conference and exhibition, Florence, Italy, Society of Petroleum Engineers, 2010.

(20) Bachu, S. Drainage and imbibition CO2/brine relative permeability curves at in situ conditions for sandstone formations in western Canada. Energy Procedia 2013,

(21) Perrin, J. C.; Benson, S. An experimental study on the influence of sub-core scale heterogeneities on $\mathrm{CO} 2$ distribution in reservoir rocks. Transp. Porous Media 2010, 82, 93-109. 
(22) Krevor, S.; Pini, R.; Zuo, L.; Benson, S. M. Relative permeability and trapping of $\mathrm{CO} 2$ and water in sandstone rocks at reservoir conditions. Water Resour. Res. 2012, 48.

(23) Zuo, L.; Krevor, S.; Falta, R. W.; Benson, S. M. An experimental study of $\mathrm{CO} 2$ exsolution and elative permeability measurements during $\mathrm{CO} 2$ saturated water depressurization. Transp. Porous Media 2012, 91, 459-478.

(24) Shi, J.-Q.; Xue, Z.; Durucan, S. Supercritical CO2 core flooding and imbibition in Tako sandstone- Influence of sub-core scale heterogeneity. Int. J. Greenhouse Gas Control 2011, 5, 75-87.

(25) Suekane, T.; Nobuso, T.; Hirai, S.; Kiyota, M. Geological storage of carbon dioxide by residual gas and solubility trapping. Int. J. Greenhouse Gas Control 2008, 2, 58-64.

(26) Chang, C.; Zhou, Q.; Xia, L.; Li, X.; Yu, Q. Dynamic displacement and non-equilibrium dissolution of supercritical $\mathrm{CO} 2$ in low-permeability sandstone: An experimental study. Int. J. Greenhouse Gas Control 2013, 14, 1-14.

(27) Akbarabadi, M.; Piri, M. Relative permeability hysteresis and capillary trapping characteristics of supercritical $\mathrm{CO} 2 /$ brine systems: an experimental study at reservoir conditions. Adv. Water Resour. 2012,

(28) Berg, S.; Oedai, S.; Ott, H. Displacement and mass transfer between saturated and unsaturated $\mathrm{CO}_{2}$-brine systems in sandstone.

Int. J. Greenhouse Gas Control 2013, 12, 478-492.

(29) Rappaport, L. A.; Leas, W. J. Properties of linear waterfloods. Trans. AIME 1953, 198, 139.

(30) Huang, D. D.; Honarpour, M. M. Capillary end effects in coreflood calculations. J. Pet. Sci. Eng. 1998, 19, 103-117.

(31) Dullien, F. A. L.; Dhawan, G. K. Bivariate pore-size distributions of some sandstones. J. Colloid Interface Sci. 1975, 52, 129-135.

(32) Wilkinson, D. Percolation effects in immiscible displacement. Phys. Rev. A 1986, 34, 1380-1391.

(33) Pini, R.; Benson, S. M. Simultaneous determination of capillary pressure and relative permeability curves from core-flooding experiments with various fluid pairs. Water Resour. Res. 2013,10.1002/ wrcr.20274.

(34) Levine, J. S. Relative permeability experiments of carbon dioxide displacing water \& their implications for carbon sequestration. Ph.D. Thesis, Columbia University, 2011.

(35) Ramakrishnan, T. S.; Cappiello, A. A new technique to measure static and dynamic properties of a partially saturated porous medium. Chem. Eng. sci. 1991, 46, 1157-1163.

(36) Fordham, E. J.; Hall, L. D.; Ramakrishnan, T. S.; Sharpe, M. R.; Hall, C. Saturation gradients in drainage of porous media: NMR imaging measurements. AIChE J. 1993, 39, 1431-1443.

(37) Pini, R.; Krevor, S.; Benson, S. M. Capillary pressure and heterogeneity for the $\mathrm{CO} 2 /$ water system in sandstone rocks at reservoir conditions. Adv. Water Resour. 2012, 38, 48-59.

(38) Lemmon, E. W.; McLinden, M. O.; Friend, D. G. Thermophysical Properties of Fluid Systems. NIST Chemistry WebBook 2010, NIST Standard Reference Database.

(39) Lide, D. R. CRC Handbook of Chemistry and Physics (Internet version 2010); CRC: Boca Raton, FL, 2010.

(40) Ramakrishnan, T. S.; Chugunov, N. Compressibility corrections to relative permeability from the non-uniform steady-state method. Chem. Eng. Sci. 2013, 73-77.

(41) Dombrowski, H. S.; Brownell, L. E. Residual equilibrium saturation of porous media. Ind. Eng. Chem. 1954, 46, 1207-1219.

(42) Berg, S.; Ott, H. Stability of CO2-brine immiscible displacement. Int. J. Greenhouse Gas Control 2012, 11, 188-203.

(43) Espinoza, D. N.; Santamarina, J. C. Water-CO2-mineral systems: Interfacial tension, contact angle, and diffusion-Implications to CO2 geological storage. Water Resour. Res. 2010, 46, W07537.

(44) Wang, S.; Edwards, I. M.; Clarens, A. F. Wettability phenomena at the co2-brine-mineral interface: Implications for geologic carbon sequestration. Environ. Sci. Technol. 2012, 47, 234-241.

(45) Broseta, D.; Tonnet, N.; Shah, V. Are rocks still water-wet in the presence of dense CO2 or H2S? Geofluids 2012, 12, 280-294.
(46) Jung, J.-W.; Wan, J. Supercritical CO2 and ionic strength effects on wettability of silica urfaces: Equilibrium contact angle measurements. Energy Fuels 2012, 26, 6053-6059.

(47) Chalbaud, C.; Lombard, J. M.; Martin, F.; Robin, M.; Bertin, H.; Egermann, P. Two phase flow properties of brine-CO2 systems in a carbonate core: Influence of wettability on Pc and kr. SPE/EAGE Reservoir Characterization and Simulation Conference 2007.

(48) Kim, Y.; Wan, J.; Kneafsey, T. J.; Tokunaga, T. K. Dewetting of silica surfaces upon reactions with supercritical $\mathrm{CO} 2$ and brine: porescale studies in micromodels. Environ. Sci. Technol. 2012, 46, 42284235.

(49) Iglauer, S.; Mathew, M.; Bresme, F. Molecular dynamics computations of brine- $\mathrm{CO} 2$ interfacial tensions and brine- $\mathrm{CO} 2$-quartz contact angles and their effects on structural and residual trapping mechanisms in carbon geo-sequestration. J. Colloid Interface Sci. 2012,

(50) Kim, T. W.; Tokunaga, T. K.; Bargar, J. R.; Latimer, M. J.;Webb, S. M. Brine film thicknesses on mica surfaces under geologic $\mathrm{CO} 2$ sequestration conditions and controlled capillary pressures. Water Resour. Res. 2013

(51) Stauffer, P. H.; Viswanathan, H. S.; Pawar, R. J.; Guthrie, G. D. A system model for geologic sequestration of carbon dioxide. Environ. Sci. Technol. 2008, 43, 565-570.

(52) Altundas, B.; Ramakrishnan, T. S.; Chugunov, N.; de Loubens, R. Retardation of $\mathrm{CO} 2$ migration due to capillary pressure hysteresis: A new CO2 trapping mechanism. Soc. Pet. Eng. J. 2011, 16, 784-794. 